\title{
E-cadherin expression is more associated with histopathological type of thyroid cancer than with the metastatic potential of tumors
}

\author{
Dorota Slowinska-Klencka ${ }^{1}$, Stanislaw Sporny ${ }^{2}$, Olga Stasikowska-Kanicka ${ }^{3}$, \\ Bozena Popowicz ${ }^{1}$, Mariusz Klencki ${ }^{1}$ \\ ${ }^{1}$ Department of Morphometry of Endocrine Glands, Chair of Endocrinology, Medical University \\ of Lodz, Poland \\ ${ }^{2}$ Department of Dental Pathomorphology, Chair of Patomorphology, Medical University of Lodz, Poland \\ ${ }^{3}$ Department of Nephropathology, Chair of Pathomorphology, Medical University of Lodz, Poland
}

\begin{abstract}
The aim of this study was to evaluate the relationship between abnormal expression of E-cadherin (E-CAD) and the extracapsular extension of tumors, lymph node involvement and the presence of metastasis in various types of thyroid cancers. Histopathological specimens of 35 benign thyroid lesions and 122 malignant tumors (papillary, follicular, poorly differentiated and undifferentiated cancers) were analyzed. E-CAD immunostaining intensity, its subcellular localization, homogeneity within lesion, and the relation of staining intensity between tumor and surrounding thyroid parenchyma were evaluated. The obtained results show that the variants of differentiated cancers with a poorer prognosis (i.e. tall cell and follicular variants of papillary cancer and widely invasive follicular cancers) present reduced intensity of E-CAD expression, its abnormal localization or heterogeneity of staining more frequently than classical papillary cancers and minimally invasive follicular cancers. However, the assessment of E-CAD expression does not allow the prediction of extrathyroidal growth of thyroid cancers. (Folia Histochemica et Cytobiologica 2012, Vol. 50, No. 4, 519-526)
\end{abstract}

Key words: thyroid, cancer, adhesion, E-cadherin

\section{Introduction}

Thyroid cancers comprise a heterogeneous group of malignant tumors. Differentiated thyroid cancers (papillary and follicular ones) are usually associated with a good prognosis, despite frequent lymph involvement in papillary cancer, or frequent distant metastases in follicular cancer. Undifferentiated cancer is a highly malignant tumor that usually leads to the patient's death. Poorly differentiated thyroid cancers occupy, both morphologically and clinically, an inter-

Correspondence address: D. Slowinska-Klencka, Department of Morphometry of Endocrine Glands, Chair of Endocrinology, Medical University of Lodz, Sterlinga St. 5, 91-425 Lodz, Poland;

tel./fax: + 48426322594 ;

e-mail: dsk@tyreo.umed.lodz.pl mediate place between the well-differentiated and undifferentiated cancers [1]. To date, in many cases, traditional histopathological examination has not been able to predict which differentiated cancers have the potential to behave aggressively. This is particularly relevant in the case of papillary microcancers. Traditional techniques are also not always useful in estimating prognosis in poorly differentiated cancers [2-3]. Thus, new methods are needed to predict the clinical course of thyroid cancers. In this respect, the attention of investigators has been drawn to the evaluation of E-cadherin expression.

Cadherins constitute a large group of proteins which are important for cell to cell adhesion, tissue organization and morphogenesis. The best known members of the superfamily of cadherins are classical cadherins - transmembrane glycoproteins with a large extracellular domain and a short intracellular 
domain which connects to the cytoskeleton through a complex of proteins among which $\alpha$ - and $\beta$-catenins play the most significant roles. E-cadherin (E-CAD) is a major classical cadherin, responsible for cell-tocell adhesion of epithelial cells. It has been postulated that the loss of normal function of cadherins may be an essential step in the progression of cancer cell dedifferentiation, leading to increased metastatic potential. Impairment of E-CAD expression has been observed to correlate with tumor invasion and metastasis in gastric and ovarian cancers, as well as other tumors [4-6]. Regulation of E-CAD gene expression has been linked to the methylation status of its promoter in several tumor models, including thyroid cancer [7].

Evaluation of E-CAD in the thyroid has focused on the relation of E-CAD expression to size of tumor, lymph nodules involvement or presence of metastasis, but the reported results are not consistent [8-15]. In only some of those reports has the expression of E-CAD been evaluated in relation to histologic subtype of papillary thyroid cancer or the pattern of local invasion in follicular cancers (minimally invasive versus widely invasive) [16-21]. Moreover, poorly differentiated thyroid cancers have been examined only in a few studies with equivocal results $[7,16,19,22]$.

The aim of this study was to evaluate E-CAD expression in thyroid tumors in relation to their histopathologic type (including some subtypes of papillary cancer), extent of tumor, lymph node involvement, and presence of metastasis.

\section{Material and methods}

Histopathological specimens of the thyroid gland were analyzed, obtained from 157 patients with various types of lesions: 35 patients with benign lesions ( 32 females and three males) and 122 with malignant tumors (107 females and 15 males). Mean age of patients with benign lesions was $49.35 \pm 9.74$ years (mean $\pm \mathrm{SD}$ ) and did not differ significantly from the mean age of patients with malignant tumors: $50.02 \pm 17.45$. All the patients had been subjected to total or subtotal thyroidectomy. The postoperative histopathological examinations were performed in the Department of Pathomorphology of M. Kopernik Regional Specialist Hospital in Lodz, Poland.

In all cases, hematoxylin and eosin-stained slides were reviewed by one of us (S.S.) and the histological patterns of the tumors were classified according to the WHO criteria. The analyzed lesions included: 18 cases of non-neoplastic thyroid tissue (nodular goiter) - NN (16 females and two males); 17 follicular adenomas - FTA ( 16 females and one male); eight follicular cancers - FTC (seven females and one male). Of these, three were minimally invasive (MI-FTC) and five widely invasive (WI-FTC). We analyzed 14 follicular cancers of oxyphilic cell type - Hürthle cancer - HTC (12 females and two males). Of these, five were minimally invasive (MI-HTC) and nine widely invasive (WIHTC). We analyzed 75 papillary cancer - PTC ( 68 females and seven males). Of these, 26 cases were of classical conventional variant - C-PTC (one male), 36 were follicular variant FV-PTC (four males), and 13 were tall cell variant TV-PTC (two males). There were 18 poorly differentiated cancers - PDTC: solid or trabecular type of poorly differentiated cancers and insular cancers (15 females and three males) and seven undifferentiated cancers - UDTD (four females and three males). The mean age of patients with NN was similar to the mean age of patients with various types of papillary cancer $(\mathrm{NN}-51.4 \pm 12.7$, C-PTC $47.7 \pm 16.1$, FV-PTC $-46.7 \pm 14.8$, TV-PTC $-50.4 \pm$ \pm 10.1 ). The mean age of patients with FTC was lower than the mean age of patients with HTC $50.6 \pm 8.5$ vs. $64.5 \pm$ $\pm 10.7(\mathrm{p}<0.05)$, but similar to the mean age of patients with FTA $45.3 \pm 13.5$. The mean age of patients with UDTC was $74.40 \pm 12.7$ years and was higher than in all other groups $(\mathrm{p}<0.05)$.

Paraffin sections were mounted onto SuperFrost slides, deparaffinized in xylene, then treated in a microwave oven in Target Retrieval Solution (Dako Cytomation, Denmark) for 30 minutes $(2 \times 5$ minutes $360 \mathrm{~W}, 2 \times 5$ minutes $180 \mathrm{~W}$, $2 \times 5$ minutes $90 \mathrm{~W}$ ) and transferred to distilled water. Endogenous peroxidase activity was blocked by $0.3 \%$ hydrogen peroxidase in distilled water for 30 minutes, and then sections were rinsed with tris-buffered saline (TBS, Dako Cytomation, Denmark) and incubated with monoclonal mouse anti-human E-cadherin antibody (Dako Cytomation, clone: NHC-38, dilution 1:200). Afterwards, EnVision+ Systems-HRP for mouse (Dako Cytomation, Denmark) prepared according to the instructions of the manufacturer were used. Visualization was performed by incubating the sections in a solution of 3,3'-diaminobenzidine (Dako Cytomation, Denmark). After washing, the sections were counter-stained with hematoxylin and coverslipped. For each antibody, and for each sample, a negative control was processed by incubation in the absence of the primary antibody. This yielded negative results in each case. For each batch, a positive control was performed using normal urothelial tissue samples and ductal breast carcinoma tissue samples. In 113 cases of thyroid tumors, adjacent normal-looking thyroid parenchyma could be identified and was therefore used as an internal control.

The intensity of staining was assessed semi-quantitatively by the evaluation of 1,000 cells in each lesion. The staining was regarded as positive in the case of membrane expression of E-CAD. The results were expressed in an ordinal scale from 0 to 4 (0: no membrane staining or staining present in $\leq 5 \%$ of cells, 1 : staining present in $6-30 \%$ of 


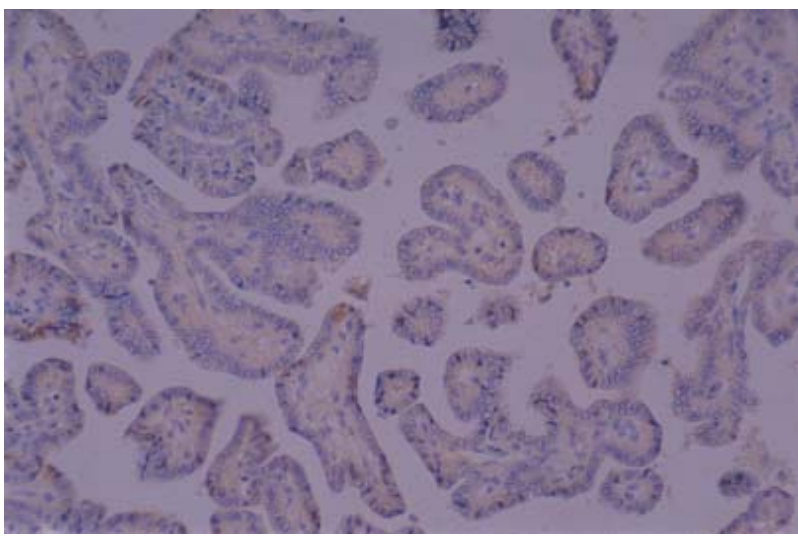

Figure 1. Papillary carcinoma - classical type - E-CAD expression markedly reduced. Magnification $\times 200$

cells, 2: in $31-60 \%$ of cells, $3:$ in $61-90 \%$ of cells, and $4:$ in more than $90 \%$ of cells). Grades of 0 and 1 were classified as markedly reduced (Figure 1), grade 2 - as reduced, and grades 3 and $4-$ as preserved (Figure 2). Apart from the intensity, other features of E-CAD immunostaining were also assessed: its subcellular localization, homogeneity within lesion, the relation of staining intensity between tumor and surrounding thyroid parenchyma. Pattern of E-CAD expression was regarded as abnormal when at least one of the following features was present: markedly reduced membrane staining (lack of membrane expression or expression in less than $30 \%$ of cells), heterogeneity of staining, or immunostaining less intensive in tumor than in its surroundings. The correlation between the extracapsular extension and size of primary tumor (T4 vs. T1-T3 and papillary microcancers vs. other papillary cancers), lymph node involvement (N0 vs. N1) or presence of metastasis (M0 vs. M1), and features of E-CAD expression were also examined.

The statistical evaluation of obtained results was performed using Spearman's coefficient, chi-square test (with Yates' correction), Mann-Whitney's U test or Student's $t$-test, where applicable.

\section{Results}

All benign lesions presented positive membrane expression of E-CAD (Table 1). In the groups of papillary cancers, the decrease in the percentage of membrane staining was $88.5 \%$ in the case of C-PTC, $83.3 \%$ - FV-PTC and $69.2 \%$ in the case of TV-PTC (TV-PTC vs. NN: $p<0.05$ ). In the group of follicular cancers, the percentage of positive staining reached $75.0 \%$ in FTC and $92.9 \%$ in HTC, and the lack of membrane expression was observed only in widely invasive cancers (WI-FTC and WI-HTC). In the PDTC group, negative staining was found in only $16.7 \%$, while in the UDTC group it was found in

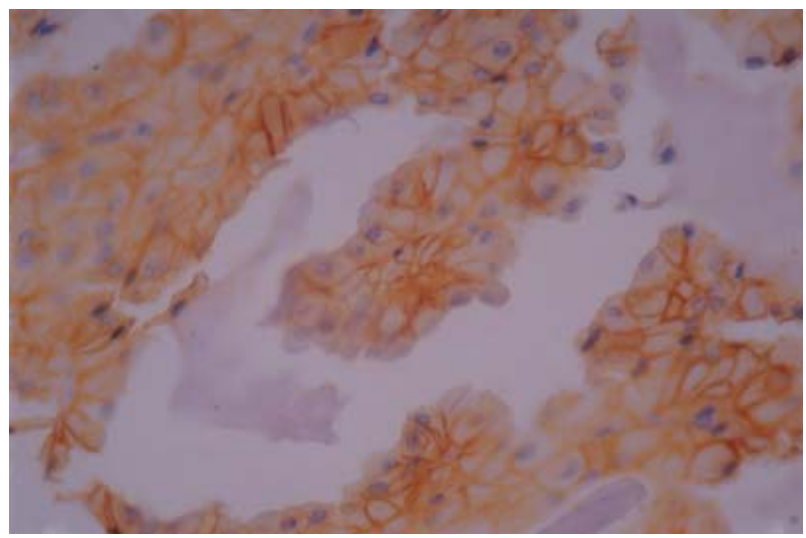

Figure 2. Papillary carcinoma - classical type - preserved membrane expression of E-CAD. Magnification $\times 500$

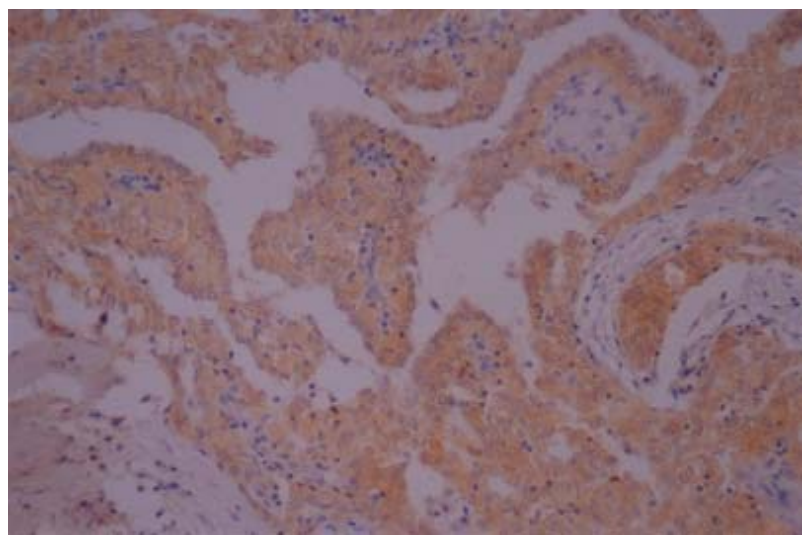

Figure 3. Papillary carcinoma - classical type - cytoplasmatic expression of E-CAD. Magnification $\times 200$

42.9\%. In the cases of negative membrane staining, the staining was positive in cytoplasm or - rarely (only in two cases of FV-PTC) - in nucleus (Figure 3).

In the groups of MI-FTC, MI-HTC and FTA, no case was found with marked reduction of E-CAD expression (positive expression in less than $30 \%$ of cells). In contrast, such a reduction was observed in all cases of UDTC - significantly more frequently than in all other groups with the exception of TV-PTC and WI-FTC. Marked reduction of the membrane E-CAD was found significantly more frequently in the groups of TV-PTC (46.2\%) and FV-PTC $(33.3 \%)$ than in the C-PTC group $(11.5 \%, \mathrm{p}<0.05$ in both cases) (Table 1).

Mean degree of E-CAD expression was the lowest in UDTC: $0.57 \pm 0.53$, mean \pm SD, (UDTC vs. FTA and C-PTC: $\mathrm{p}<0.0001$; UDTC vs. other groups: $\mathrm{p}<0.005$ ) (Table 1). The highest mean degree of E-CAD expression $(>3)$ was noted in follicular neoplasms (FTA: $3.24 \pm 0.83$, MI-FTC: $3.33 \pm 0.58$, 
Table 1. Characteristics of E-CAD in various thyroid tumors and non-neoplastic thyroid tissue $(*$ in some cases missing surrounding tissues in the specimen)

\begin{tabular}{|l|c|c|c|c|c|c|}
\hline \multirow{2}{*}{ Type of tumor } & \multicolumn{5}{|c|}{ Characteristics of E-CAD expression } \\
\cline { 2 - 7 } & $\begin{array}{c}\text { Positive } \\
\text { membrane } \\
\text { staining }\end{array}$ & $\begin{array}{c}\text { Mean degree } \\
\pm \text { SD }\end{array}$ & $\begin{array}{c}\text { Intensity markedly } \\
\text { reduced } \\
(\mathbf{0}-\mathbf{1} \text { degree })\end{array}$ & $\begin{array}{c}\text { Intensity preserved } \\
(>\mathbf{2} \text { degree })\end{array}$ & $\begin{array}{c}\text { Homogenous } \\
\text { expression }\end{array}$ & $\begin{array}{c}\text { Expression in } \\
\text { tumor similar } \\
\text { to surrounding } \\
\text { tissues }\end{array}$ \\
\hline NN & $18 / 18(100.0 \%)^{\mathrm{df}}$ & $2.56 \pm 0.92^{\mathrm{b}}$ & $2 / 18(11.10 \%)^{\mathrm{afk}}$ & $12 / 18(66.7 \%)^{\mathrm{bf}}$ & $4 / 18(22.2 \%)$ & - \\
\hline C-PTC & $23 / 26(88.5 \%)$ & $2.73 \pm 1.28^{\mathrm{afl}}$ & $3 / 26(11.5 \%)^{\mathrm{afk}}$ & $16 / 26(61.5 \%)^{\mathrm{d}}$ & $12 / 23(52.2 \%)$ & $13 / 20^{*}(65.0 \%)$ \\
\hline FV-PTC & $30 / 36(83.3 \%)$ & $2.22 \pm 1.42^{\mathrm{b}}$ & $12 / 36(33.3 \%)^{\mathrm{b}}$ & $18 / 36(50.0 \%)^{\mathrm{c}}$ & $16 / 30(53.3 \%)$ & $27 / 34^{*}(79.4 \%)^{\mathrm{c}}$ \\
\hline TV-PTC & $9 / 13(69.2 \%)$ & $1.61 \pm 1.39^{\mathrm{b}}$ & $6 / 13(46.2 \%)$ & $4 / 13(30.8 \%)$ & $2 / 9(22.2 \%)^{\mathrm{hj}}$ & $6 / 11^{*}(54.5 \%)$ \\
\hline FTA & $17 / 17(100.0 \%)^{\mathrm{c}}$ & $3.24 \pm 0.83^{\mathrm{al}}$ & $0 / 17(0.0 \%)^{\mathrm{ack}}$ & $13 / 17(76.5 \%)^{\mathrm{b}}$ & $14 / 17(82.4 \%)$ & $16 / 17(94.1 \%)^{\mathrm{c}}$ \\
\hline MI-FTC & $3 / 3(100.0 \%)$ & $3.33 \pm 0.58^{\mathrm{b}}$ & $0 / 3(0.0 \%)^{\mathrm{d}}$ & $3 / 3(100.0 \%)^{\mathrm{c}}$ & $2 / 3(66.7 \%)$ & $2 / 2^{*}(100.0 \%)$ \\
\hline WI-FTC & $3 / 5(60.0 \%)$ & $1.80 \pm 1.79^{\mathrm{b}}$ & $2 / 5(40.0 \%)$ & $2 / 5(40.0 \%)$ & $3 / 3(100.0 \%)$ & $2 / 2^{*}(100.0 \%)$ \\
\hline MI-HTC & $5 / 5(100.0 \%)$ & $3.20 \pm 0.84^{\mathrm{b}}$ & $0 / 5(0.0 \%)^{\mathrm{b}}$ & $4 / 5(80.0 \%)^{\mathrm{c}}$ & $3 / 5(60.0 \%)$ & $3 / 3^{*}(100.0 \%)$ \\
\hline WI-HTC & $8 / 9(88.9 \%)$ & $3.11 \pm 1.27^{\mathrm{b}}$ & $1 / 9(11.1 \%)^{\mathrm{b}}$ & $8 / 9(88.9 \%)^{\mathrm{b}}$ & $7 / 8(87.5 \%)$ & $5 / 5^{*}(100.0 \%)$ \\
\hline PDTC & $15 / 18(83.3 \%)$ & $2.11 \pm 1.28^{\mathrm{b}}$ & $5 / 18(27.8 \%)^{\mathrm{c}}$ & $8 / 18(44.4 \%)$ & $3 / 15(20.0 \%)^{\mathrm{gj}}$ & $12 / 15^{*}(80.0 \%)^{\mathrm{d}}$ \\
\hline UDTC & $4 / 7(57.1 \%)$ & $0.57 \pm 0.53$ & $7 / 7(100.0 \%)$ & $0 / 7(0.0 \%)$ & $0 / 4(0.0 \%)^{\mathrm{hj}}$ & $0 / 4^{*}(0.0 \%)$ \\
\hline
\end{tabular}

$\mathrm{a}-\mathrm{p}<0.0001$ vs. UDTC; $\mathrm{b}-\mathrm{p}<0.005$ vs. UDTC; $\mathrm{c}-\mathrm{p}<0.01$ vs. UDTC; $\mathrm{d}-\mathrm{p}<0.05$ vs. UDTC; $\mathrm{e}-\mathrm{p}<0.01$ vs. TV-PTC; $\mathrm{f}-\mathrm{p}<0.05$ vs. TV-PTC; $\mathrm{g}-\mathrm{p}<0.005$ vs. FTA; $\mathrm{h}-\mathrm{p}<0.01$ vs. FTA; $\mathrm{i}-\mathrm{p}<0.01$ vs. WI-HTC; $\mathrm{j}-\mathrm{p}<0.05$ vs. WI-HTC; $\mathrm{k}-\mathrm{p}<0.05$ vs. FV-PTC; $1-\mathrm{p}<0.05$ vs. PDTC

MI-HTC: $3.20 \pm 0.84$, WI-HTC: $3.11 \pm 1.27)$ with the exception of WI-FTC $(1.80 \pm 1.79)$. No difference was found in mean degree of E-CAD expression between NN (2.56 \pm 0.92$)$ and any variant of PTC, but it was found between C-PTC and TV-PTC $(2.73 \pm$ \pm 1.28 vs. $1.61 \pm 1.39, p<0.05)$. Mean expression of E-CAD in PDTC $(2.11 \pm 1.28)$ was lower than in FTA and C-PTC ( $\mathrm{p}<0.05$ in both cases) and comparable with FV-PTC ( $2.22 \pm 1.42)$, TV-PTC and WI-FTC.

Preserved E-CAD expression - the presence of staining in more than $60 \%$ of cells - was observed in more than $60 \%$ of $\mathrm{NN}$ and C-PTC cases, in $50.0 \%$ of FV-PTC, but in only $30.8 \%$ of TV-PTC (NN vs. TV-PTC: $p<0.05)$. In the group of follicular cancers, it was found in at least $80 \%$ of cases of MI-FTC, MI-HTC and WI-HTC - thus with a frequency similar to that observed in the group of FTA $(76.5 \%)$ - but only in $40.0 \%$ of WI-FTC. In the PDTC group, preserved expression E-CAD was noted in $44.4 \%$ of cases, and in the UDTC group - in none of the cases (UDTC vs. NN, FTA and WI- HTC: $p<0.005$; UDTC vs. MI-HTC, MI-FTC, FV-PTC and C-PTC: $\mathrm{p}<0.05)$ (Table 1).

In the UDTC, PDTC and TV-PTC groups, the immunostaining was homogenous twice or thrice less frequently than in the groups of other cancers (UDTC, PDTC and TV-PTC together vs. other cancers together: $\mathrm{p}<0.0005)$. On the other hand, in the majority of cases (77.8\%), the NN group presented non-homogenous immunostaining against E-CAD ( $p<0.005$ vs. FTA).

E-CAD expression in all cases of follicular cancers was equal to or higher than that observed in surrounding tissues. In all four cases of UDTC, in which there were surrounding tissues present in the specimen and in some cases of other cancers, the staining in the surrounding tissues was higher than in the tumor (in $29.2 \%$ of PTC, and in $20.0 \%$ of PDTC).

The pattern of E-CAD expression was abnormal in $100.0 \%$ of UDTC, $88.9 \%$ of PDTC and $84.6 \%$ of TV-PTC - significantly more frequently than in the FTA group - 17.6\% (3/17) (UDTC and TV-PTC vs. FTA: $p<0.005$; PDTC vs. FTA: $p<0.0001)$. In all other groups of papillary cancers and in the WI-FTC group, abnormal pattern of E-CAD expression was observed in 55-60\% of cases. In the FTA, MI-FTC, and MI-HTC groups, abnormal pattern of E-CAD expression was in each case connected with heterogeneity of staining only. Similarly, in the majority of cases in the NN group, abnormal E-CAD expression was related to heterogeneity of staining (12 of 14 cases with abnormal pattern). In both groups of widely invasive follicular cancers (FTC and HTC), cases with lowered expression or lack of membrane localization were also observed. In the PTC, PDTC and UDTC cases, all analyzed types of abnormality were found 
Table 2. Abnormal pattern of E-CAD expression in various thyroid tumors; comparison of E-CAD expression in cancers limited to the thyroid (T1-3, N0, M0) and in tumors showing extrathyroid growth (N1 or M1 or T4). Abnormal pattern of E-CAD - at least one of the following features: lack of immunostaining or staining positive in less than $30 \%$ of cells, lack of membrane localization, heterogeneity of staining, or immunostaining less intensive in tumor than in its surroundings

\begin{tabular}{|l|c|c|c|c|c|c|}
\hline \multirow{2}{*}{ Type of tumor } & \multicolumn{3}{|c|}{ Abnormal pattern of E-CAD expression } & \multicolumn{2}{c|}{$\begin{array}{c}\text { Abnormal pattern of E-CAD expression, } \\
\text { except for heterogeneity of staining }\end{array}$} \\
\cline { 2 - 7 } & All TNMs & $\begin{array}{c}\text { N0 and M0 } \\
\text { and T<4 }\end{array}$ & $\begin{array}{c}\text { N1 or M1 } \\
\text { or T4 }\end{array}$ & All TNMs & $\begin{array}{c}\text { N0 and M0 } \\
\text { and T<4 }\end{array}$ & $\begin{array}{c}\text { N1 or M1 } \\
\text { or T4 }\end{array}$ \\
\hline NN & $14 / 18(77.8 \%)^{\text {bd }}$ & - & - & $2 / 18(11.1 \%)$ & - & - \\
\hline C-PTC & $15 / 26(57.7 \%)$ & $8 / 12(66.7 \%)$ & $7 / 14(50.0 \%)$ & $8 / 26(30.7 \%)$ & $3 / 12(25.0 \%)$ & $5 / 14(35.7 \%)$ \\
\hline FV-PTC & $20 / 36(55.5 \%)$ & $14 / 25(56.0 \%)$ & $6 / 11(54.5 \%)$ & $12 / 36(33.3 \%)$ & $10 / 25(40.0 \%)$ & $2 / 11(18.2 \%)$ \\
\hline TV-PTC & $11 / 13(84.6 \%)^{\text {bd }}$ & $3 / 3(100.0 \%)$ & $8 / 10(80.0 \%)$ & $7 / 11(63.6 \%)$ & $2 / 3(66.7 .0 \%)$ & $5 / 10(50.0 \%)$ \\
\hline FTA & $3 / 17(17.6 \%)$ & - & - & $0 / 17(0.0 \%)$ & - & - \\
\hline MI-FTC & $1 / 3(33.3 \%)$ & $1 / 3(33.3 \%)$ & $0 / 0(0.0 \%)$ & $0 / 3(0.0 \%)$ & $0 / 3(0.0 \%)$ & $0 / 0(0.0 \%)$ \\
\hline WI-FTC & $3 / 5(60.0 \%)$ & $1 / 1(100.0 \%)$ & $2 / 4(50.0 \%)$ & $2 / 5(40.0 \%)$ & $1 / 1(100.0 \%)$ & $1 / 4(25.0 \%)$ \\
\hline MI-HTC & $2 / 5(40.0 \%)$ & $2 / 4(50.0 \%)$ & $0 / 1(0.0 \%)$ & $0 / 5(0.0 \%)$ & $0 / 4(0.0 \%)$ & $0 / 1(0.0 \%)$ \\
\hline WI-HTC & $2 / 9(22.2 \%)$ & $2 / 3(66.7 \%)$ & $0 / 6(0.0 \%)$ & $1 / 9(11.1 \%)$ & $1 / 3(33.3 \%)$ & $0 / 6(0.0 \%)$ \\
\hline PDTC & $16 / 18(88.9 \%)^{\mathrm{ace}}$ & $8 / 9(88.9 \%)$ & $8 / 9(88.9 \%)$ & $7 / 18(38.9 \%)$ & $4 / 9(44.4 \%)$ & $3 / 9(33.3 \%)$ \\
\hline UDTC & $7 / 7(100.0 \%)^{\mathrm{b}}$ & $1 / 1(100.0 \%)$ & $6 / 6(100.0 \%)$ & $7 / 7(100.0 \%)$ & $1 / 1(100.0 \%)$ & $6 / 6(100.0 \%)$ \\
\hline
\end{tabular}

$\mathrm{a}-\mathrm{p}<0.0001$ vs. FTA; $\mathrm{b}-\mathrm{p}<0.005$ vs. FTA; $\mathrm{c}-\mathrm{p}<0.005$ vs. WI-HTC; $\mathrm{d}-\mathrm{p}<0.05$ vs. WI-HTC; $-\mathrm{p}<0.05$ vs. FV-PTC

(Table 2). If heterogeneity of staining was excluded from the set of features indicating abnormal pattern of E-CAD expression, then the percentage of such patterns dropped to 0 in the groups of FTA, MI-FTC, MI-HTC, and was lowered in the NN and PDTC groups, but remained unchanged in the UDTC group (Table 2).

There was no correlation between the abnormal pattern of E-CAD expression and features of extrathyroid growth of cancers (Table 2). No relation was found even when each examined feature of E-CAD expression was analyzed separately and compared to the extent of primary tumor (T4 vs. T1-T3), lymph node involvement (N1 vs. N0) and presence of metastasis (M1 vs. M0). Abnormal pattern of E-CAD expression was observed in $44.4 \%$ of papillary microcancers $(4 / 9)$ and in $63.6 \%$ of other papillary cancers (42/66), p - NS.

\section{Discussion}

Quantitative evaluation of E-CAD immunoexpression is difficult. Heterogeneity of staining in various regions of tumors and the lack of an unequivocal definition for the reduction of staining intensity make results from different centers very difficult to compare. Some investigators prefer to use only a descriptive scale of staining intensity, e.g.: very weak, mod- erate, or strong expression $[8,16,17,20]$. Others accept a well defined limit of the percentage of positively stained cells to distinguish reduction of E-CAD expression. Scheumman et al. (1995) set such a limit at $30 \%$ of positive cells in tumor [9]. Naito et al. (2001) treated E-CAD expression as preserved if more than $50 \%$ of cells were stained, and Kato et al. (2002) raised that limit to $70 \%$ of tumor cells $[12,18]$. The highest limit was used by Brecelj et al. (2005), who defined reduced E-CAD expression as $<90 \%$ of cells showing membrane positivity [19].

Undoubtedly, these discrepancies are at least partially responsible for some contradictions between the published reports. Another cause is related to the lack of distinguishing between subtypes of papillary cancer and follicular cancers, as well as to differences in classification of poorly differentiated thyroid cancers.

In the present study, we decided to assume $<30 \%$ of positively stained cells as the limit for markedly reduced expression, and $>60 \%$ as the limit for preserved expression. Additionally, the study assessed not only the staining intensity but also the homogeneity of staining and the relation between intensity of E-CAD staining in a tumor and its surroundings. Various subtypes of papillary and follicular cancers were analyzed separately, and the examined features of E-CAD expression were compared to the intensity of 
tumor growth (size and extent of the tumor, lymph node involvement and presence of distant metastases).

We found, in accord with many other reports, that E-CAD expression is most reduced in UDTC $[7,8$, 16, 23-25].

In PTC, E-CAD expression was reduced more frequently in non-classical variants - TV-PTC and FV-PTC - than in classical ones. Also, the heterogeneity of the expression or its relocation from membrane to the cytoplasm or nucleus was observed in TV-PTC more than twice as frequently as in FV-PTC and C-PTC. Rocha et al. (2001) showed relocation to the cytoplasm in a diffuse sclerosing variant of PTC in contrast to classical PTC. However, no detailed report has yet been published on alterations in E-CAD expression in TV-PTC [17].

In FTC and HTC, E-CAD expression in terms of intensity of staining, membranous localization and homogeneity was similar to that observed in benign lesions (especially FA) and differed significantly from that seen in PTC. This is concordant with the reports by other authors $[7,8,12,16,20,23,26,27]$. Only von Wasilewski et al. (1997) did not find differences in E-CAD expression between PTC and FTC [11]. We found some cases of abnormal E-CAD localization and marked reduction of its expression only in the groups of WI-FTC and WI-HTC. Brecelj et al. (2005) reported that reduced E-CAD expression was significantly associated with widely invasive growth of FTC but was not related to the size of tumor, which is concordant with our data [19].

In PDTC, marked reduction of E-CAD expression was observed in only $27.8 \%$ of cases, but abnormal pattern of E-CAD expression (including also heterogeneity of staining, non-membranous localization or reduced intensity compared to the surrounding parenchyma) was present in $88.9 \%$ of cases. The reports on PDTC are much less unequivocal. Rocha et al. (2003) found that none of 17 cases of PDTC displayed membranous immunoreactivity [22]. Only two cases showed E-CAD expression, but it was aberrantly localized (cytoplasmatic or granular in the apical pole of the cells). In contrast, Choi et al. (2005) observed expression of E-CAD in 58\% of cases of PDTC compared to no cases of UDTC, 33\% of PTCs, $80 \%$ of FTCs, and $83 \%$ of HTCs [20]. It should be kept in mind that 'poorly differentiated cancer' is an umbrella designation of a heterogeneous group of thyroid cancers. According to the WHO classification, a tumor is diagnosed as PDTC when any of insular, trabecular, or solid histological patterns is recognized in the majority of the tumor along with an infiltrative pattern of growth, necrosis, and obvious vascular invasion [28]. However, these criteria are difficult to employ in daily practice as there is no clear-cut separation between PDTC, the solid variant of PTC and well-differentiated FTC with predominantly solid/trabecular growth pattern $[29,30]$. It is also important that within a single lesion both strong membrane positivity in the well-differentiated areas, and absence of E-CAD expression in undifferentiated areas of PDTC, may be observed [16]. This constitutes an additional problem regarding the interpretation of the results of immunostaining against E-CAD, which is itself difficult to quantify. This problem is well illustrated by the exclusion of heterogeneity from the criteria of abnormal pattern of E-CAD expression, which leads to a marked reduction in the number of PDTC cases with abnormal E-CAD expression. Interesting$1 y$, the expression of heterogeneity was also very common in non-neoplastic thyroid tissue. This was probably caused by the presence of hyperplastic nodules in nodular goiter, and not only normal thyroid tissue in that group.

Although we found abnormal E-CAD expression to be more frequent in cancers showing a poorer prognosis - UDTC, PDTC, TV-PTC and WI-FTC - no significant relation was found between abnormalities in E-CAD expression and the features of extrathyroidal growth of cancers (extracapsular invasion, lymph nodes involvement or metastases). No difference was shown in any of the analyzed features of E-CAD expression between papillary microcancers with other papillary cancers. Similarly, Kapran et al. (2002) did not find a correlation between E-CAD immunoreactivities and local invasion or lymphatic spread of papillary cancers [13]. Soares et al (1997), as well as Nikiel et al. (2006), reported that PTC which had lymph node involvement did not disclose any major difference in intensity of immunoreactivity and percentage of immunoreactive cells [14, 16]. Erdem et al. (2011) did not find any difference in E-CAD expression in relation to the size of tumor, capsule invasion or metastases [15]. On the other hand, Naito et al. (2001) found that E-CAD expression was preserved significantly less often in papillary cancer with lymph node metastasis than in node-negative cases, but E-CAD expression did not correlate significantly with other clinical factors including tumor diameter, extracapsular tumor extension or histological differentiation [12]. These and our results are thus in contrast to those reported by Brabant, von Wasilewski, Scheumman, Walgenbach and Batistatou [8-11, 31]. Brabant et al. (1993) observed lower or undetectable E-CAD immunoreactivity in tumors presenting lymph node metastases, distant metastases or local recurrence [8]. von Wasilewski et al. (1997) found that the lack of E-CAD expression was more frequent among 
T3-T4 cancers compared to T1-T2 tumors and among cancers with distant metastases compared to M0 tumors [11]. Scheumman et al. (1995) and Walgenbach et al. (1998) reported that reduced (below $30 \%$ in the study by Scheumman and $<20 \%$ - in Walgenbach's study) or negative E-CAD expression was associated with advanced $\mathrm{T}$ categories of the primary tumors and higher rates of lymph node involvement and distant metastasis [9, 10]. Batistatou et al. (2008) found that the reduction of E-CAD expression in papillary microcancers was smaller than in other papillary cancers [31]. Our data did not confirm those observations.

In conclusion, the assessment of E-CAD expression does not enable the prediction of extrathyroidal growth of thyroid cancers. However, there are significant differences in the expression of E-CAD between various subtypes of papillary cancer. Variants of PTC with a poorer prognosis - particularly the tall cell variant - present reduced E-CAD expression, its abnormal localization or heterogeneity of staining more frequently than classical PTC. Similarly, some widely invasive follicular thyroid cancers show abnormal pattern of E-CAD expression in contrast to minimally invasive follicular cancers, which are similar to follicular adenomas in this respect. Abnormal E-CAD expression is most commonly observed in undifferentiated thyroid cancers.

In our opinion, it should not be surprising that some aggressively growing thyroid cancers show E-CAD expression while some others, with a benign clinical course, present markedly reduced or abnormal expression of this protein. Obviously, E-CAD is not the only protein on which cell-to-cell adhesion relies. In fact, it has been shown that thyroid follicular cells can maintain adhesion in the total absence of E-CAD [32]. It is probable that disruption of other proteins can lead to the loss of cell-to-cell adhesion, even in the presence of normally expressed E-CAD.

\section{References}

1. Gharib H, Papini E, Paschke R et al. AACE/AME/ETA Task Force on Thyroid Nodules. Endocr Pract. 2010;16(Suppl. 1):1-43.

2. Akslen LA, LiVolsi VA. Prognostic significance of histologic grading compared with subclassification of papillary thyroid cancer. Cancer. 2000;88:1902-1908.

3. Hiltzik D, Carlson DL, Tuttle RM et al. Poorly differentiated thyroid cancers defined on the basis of mitosis and necrosis: a clinicopathologic study of 58 patients. Cancer. 2006;106:1286-1295.

4. Bracke ME, van Roy FM, Mareel MM. The E-cadherin/catenin complex in invasion and metastasis. Curr Top Microbiol Immunol. 1996;213:123-161.

5. Sawada K, Mitra AK, Radjabi AR et al. Loss of E-cadherin promotes ovarian cancer metastasis via alpha 5-integrin, which is a therapeutic target. Cancer Res. 2008;68:2329-2339.

6. Zhong XY, Zhang LH, Jia SQ et al. Positive association of up-regulated Cripto-1 and down-regulated E-cadherin with tumour progression and poor prognosis in gastric cancer. Histopathology. 2008;52:560-568.

7. Graff JR, Greenberg VE, Herman JG et al. Distinct patterns of E-cadherin $\mathrm{CpG}$ island methylation in papillary, follicular, Hurthle's cell, and poorly differentiated human thyroid cancer. Cancer Res. 1998;58:2063-2066.

8. Brabant G, Hoang-Vu C, Cetin Y et al. E-cadherin: a differentiation marker in thyroid malignancies. Cancer Res. 1993;53: 4987-4993.

9. Scheumman GF, Hoang-Vu C, Cetin Y et al. Clinical significance of E-cadherin as a prognostic marker in thyroid cancers. J Clin Endocrinol Metab. 1995;80:2168-2172.

10. Walgenbach S, Sternheim E, Bittinger F, Görges R, Andreas $\mathrm{J}$, Junginger T. Prognostic value of e-cadherin in papillary thyroid cancer. Chirurg. 1998;69:186-190.

11. von Wasielewski R, Rhein A, Werner M et al. Immunohistochemical detection of E-cadherin in differentiated thyroid cancers correlates with clinical outcome. Cancer Res. 1997;57:2501-2507.

12. Naito A, Iwase H, Kuzushima T, Nakamura T, Kobayashi S. Clinical significance of E-cadherin expression in thyroid neoplasms. J Surg Oncol. 2001;76:176-180.

13. Kapran Y, Ozbey N, Molvalilar S, Sencer E, Dizdaroglu F, Ozarmagan S. Immunohistochemical detection of E-cadherin, alpha- and beta-catenins in papillary thyroid cancer. J Endocrinol Invest. 2002;25:578-585.

14. Nikiel B, Chekan M, Jaworska M, Jarzab M, Maksymiuk B, Lange D. Expression of the selected adhesive molecules (cadherin E, CD44, LGAL3 and CA50) in papillary thyroid cancer. Endokrynol Pol. 2006;57:326-335.

15. Erdem H, Gündogdu C, Sipal S. Correlation of E-cadherin, VEGF, COX-2 expression to prognostic parameters in papillary thyroid carcinoma. Exp Mol Pathol. 2011;90:312-317.

16. Soares P, Berx G, van Roy F, Sobrinho-Simőes M. E-cadherin gene alterations are rare events in thyroid tumors. Int J Cancer. 1997;70:32-38.

17. Rocha AS, Soares P, Seruca R et al. Abnormalities of the E-cadherin/catenin adhesion complex in classical papillary thyroid cancer and in its diffuse sclerosing variant. J Pathol. 2001;194:358-366.

18. Kato N, Tsuchiya T, Tamura G, Motoyama T. E-cadherin expression in follicular cancer of the thyroid. Pathol Int. 2002;52:13-18.

19. Brecelj E, Frković Grazio S, Auersperg M, Bracko M. Prognostic value of E-cadherin expression in thyroid follicular cancer. Eur J Surg Oncol. 2005;31:544-5485.

20. Choi YL, Kim MK, Suh JW et al. Immunoexpression of HBME-1, high molecular weight cytokeratin, cytokeratin 19, thyroid transcription factor-1, and E-cadherin in thyroid cancers. J Korean Med Sci. 2005;20:853-859.

21. Ito Y, Hirokawa M, Fukushima M et al. Prevalence and Prognostic Significance of Poor Differentiation and Tall Cell Variant in Papillary Cancer in Japan. World J Surg. 2008;32:1535-1545.

22. Rocha AS, Soares P, Fonseca E, Cameselle-Teijeiro J, Oliveira MC, Sobrinho-Simőes M. E-cadherin loss rather than beta-catenin alterations is a common feature of poorly differentiated thyroid cancers. Histopathology. 2003;42:580-587.

23. Serini G, Trusolino L, Saggiorato E et al. Changes in integrin and E-cadherin expression in neoplastic versus normal thyroid tissue. J Natl Cancer Inst. 1996;88:442-449.

24. Cerrato A, Fulciniti F, Avallone A, Benincasa G, Palombini L, Grieco M. Beta- and gamma-catenin expression in thyroid cancers. J Pathol. 1998;185:267-272. 
25. Sato H, Ino Y, Miura A et al. Dysadherin: expression and clinical significance in thyroid cancer.J Clin Endocrinol Metab. 2003;88:4407-4412.

26. Huang SH, Wu JC, Chang KJ, Liaw KY, Wang SM. Expression of the cadherin-catenin complex in well-differentiated human thyroid neoplastic tissue. Thyroid. 1999;9:1095-1103.

27. Ozolins A, Narbuts Z, Strumfa I, Volanska G, Gardovskis J. Diagnostic utility of immunohistochemical panel in various thyroid pathologies. Langenbecks Arch Surg. 2010;395: 885-891.

28. DeLellis RA, Lloyd R, Heitz PU. (eds) WHO Classification of Tumors, Pathology and Genetics - Tumors of Endocrine Organs. Lyon: IARC Press, 2004.

29. Sobrinho-Simőes M, Sambade C, Fonseca E, Soares P. Poorly differentiated cancers of the thyroid gland: a review of the clinicopathologic features of a series of 28 cases of a heterogeneous, clinically aggressive group of thyroid tumors. Int J Surg Pathol. 2002;10:123-131.

30. Volante M, Collini P, Nikiforov YE et al. Poorly differentiated thyroid cancer: the Turin proposal for the use of uniform diagnostic criteria and an algorithmic diagnostic approach. Am J Surg Pathol. 2007;31:1256-1264.

31. Batistatou A, Charalabopoulos K, Nakanishi Y et al. Differential expression of dysadherin in papillary thyroid carcinoma and microcarcinoma: correlation with E-cadherin. Endocr Pathol. 2008;19:197-202.

32. Calě $G$, Zannini M, Rubini $P$ et al. Conditional inactivation of the E-cadherin gene in thyroid follicular cells affects gland development but does not impair junction formation. Endocrinology. 2007;148:2737-2746.

Submitted: 27 October, 2011 Accepted after reviews: 8 November, 2012 